\title{
Valoración de la docencia clínica en tercer curso del Grado de Medicina. Estudio secuencial de cinco cursos
}

\author{
Josep M. Grau, Emilia Antúnez, Albert Cobos
}

Introducción. Existen pocos estudios que de forma selectiva valoren la docencia clínica de una determinada asignatura. En este artículo se presenta la experiencia en docencia clínica de la asignatura 'Semiología General y Propedéutica Clínica' en tercer curso del Grado de Medicina en la Facultad de Medicina de la Universitat de Barcelona.

Materiales y métodos. Se han analizado los cursos 2011-2012, 2012-2013 y 2013-2014, representando un total de cinco 'cursos', ya que la asignatura es semestral y se imparte dos veces en cada curso académico. El período de docencia clínica de la asignatura es de siete semanas (28 días) y el número de alumnos oscila entre cuatro y ocho por cada una de las siete unidades/centros distintos. A su llegada se les informa de los objetivos de la estancia clínica y al final del período se les invita a cumplimentar una encuesta anónima y voluntaria en la que valoran distintos ítems.

Resultados. Se han recogido 477 encuestas (95\%). Se constató una muy buena valoración global del período de docencia clínica (mediana: 5; primer cuartil: 4, en escala de 1 a 5), sin variaciones significativas entre los distintos períodos evalua$\operatorname{dos}(p=0,658)$.

Conclusiones. La docencia clínica en esta asignatura está muy bien valorada de forma global, sin detectar variaciones relevantes en los cinco cursos analizados.

Palabras clave. Docencia clínica. Grado de Medicina.

\section{Clinical skills training in the third course of Medicine. Sequential evaluation of five cycles}

Introduction. There are very few studies regarding the clinical skills training of a particular matter. In the present study the experience in clinical training in the matter 'Semiology and Clinical Propedeutics' from the Faculty of Medicine in the University of Barcelona is presented. The results of surveys from five consecutive cycles are compared.

Materials and methods. The period of study represents five cycles (2011-2014) since the matter is developed twice a year. The clinical period training in the matter spent seven weeks (28 days) and the number of students for each of the seven different facilities ranged from 4-8. When arriving, the students are informed about the objectives to be achieved through their training period, and at the end they are asked about the voluntary and anonymous survey complementation.

Results. A total of $95 \%(n=477)$ of the requested surveys were collected. A very good global qualification was assessed (median: 5; first quartile: 4 , in 1-5 scale), without significant differences among the different evaluated periods of time $(p=0.658)$.

Conclusions. Clinical skills training in this matter is considered near excellent without significant variations through the five consecutive periods analyzed.

Key words. Clinical skills. Medicine degree.

\section{Introducción}

Existe un interés creciente por la calidad educativa, siendo la evaluación de la docencia un elemento importantísimo para analizar la calidad de las instituciones educativas, dado que el objetivo primordial de la enseñanza universitaria es, a grandes rasgos, la consecución de la óptima preparación de los profesionales del futuro. En este sentido, en 2011, Baños et al [1] describieron con detalle y específica- mente los requisitos académicos, los objetivos, las competencias que deben alcanzarse y el sistema de evaluación. Este tipo de evaluación tiene su origen formal en torno a los años veinte del pasado siglo en Estados Unidos, donde hoy día es una práctica muy asentada en casi la totalidad de sus universidades [2] y desde entonces se ha extendido por los cinco continentes [3-8]. Actualmente, la universidad se encuentra inmersa en un proceso de cambio que viene marcado, por un lado, por la legislación
Departamento de Medicina (J.M. Grau, E. Antúnez); Departamento de Salud Pública (A. Cobos). Facultad de Medicina. Universitat de Barcelona. Barcelona, España.

Correspondencia:

Dr. Josep M. Grau Junyent. Departamento de Medicina. Facultad de Medicina. Universitat de Barcelona. Casanova, 143. E-08036 Barcelona.

E-mail:

jmgrau@clinic.ub.es

Agradecimientos:

A los miembros del equipo docente que han participado como tutores y profesores clínicos en cada uno de los centros/unidades donde se han desarrollado las prácticas clínicas: X. Bosch, L. Buisán, M. Camafort, F. Cardellach, P. Castro, A. Coca, J. Delás, M.C. Cid, B. Coll-Vinent, A. de la Sierra, R. Estruch,

J. Fernàndez-Solà, I. de Lecuona, M. Larrousse, A. López-Soto, J. Martínez-Montauti, F. Masanés, J.M. Nicolás, M. Navarro, E. Sacanella, C. Sierra, M. Torres-Salinas, A. Trilla, J. Villalta. Al profesor J.E. Baños, por su lectura crítica del artículo y por sus consejos en la discusión del mismo.

Conflicto de intereses: No declarado.

Competing interests: None declared.

(c) 2015 FEM 
Tabla I. Objetivos de la docencia clínica.

Aprender a obtener una historia clínica completa (anamnesis)

Saber hacer una exploración física completa que incluye: constantes vitales, aspecto general del paciente, piel y mucosas, existencia de adenopatías, exploración mamaria, exploración de los aparatos: respiratorio, cardiocirculatorio, del abdomen, genital, aparato locomotor y sistema nervioso

Conocer y utilizar la informática para consultar datos de la historia y de las exploraciones complementarias

Saber interpretar una radiografía de tórax y otras exploraciones de imagen

Saber interpretar un electrocardiograma, con reconocimiento de ritmo sinusal, bloqueos, crecimientos e hipertrofias de cavidades cardíacas y signos de isquemia

Saber interpretar los resultados de lo que se considera una analítica general básica

Saber comunicarse con el paciente y su entorno, de forma clara, ordenada, eficiente y respetuosa

Saber relacionarse con el resto del equipo asistencial

Conocer los aspectos éticos de la relación asistencial

Asistir a un taller de habilidades clínicas con el profesor clínico un día durante cuatro horas, donde se les enseña, con un maniquí que lleva incorporado un programa, a reconocer los ruidos respiratorios, los ruidos cardíacos, acompañados de un vídeo donde se les explica las diferentes patologías valvulares con ilustraciones anatómicas y de ecocardiograma, además de la exploración prostática, la exploración mamaria y la toma de presión arterial

Asistir a urgencias realizando una guardia de 12 horas consecutivas, con un tutor asignado a cada alumno

Asistir durante dos mañanas al dispositivo de consulta externa (unidad de diagnóstico rápido y consulta externa general de medicina interna) con su tutor

(Ley Orgánica de Universidades, 2001) y su posterior modificación (2007), y por otro, la adaptación de nuestros títulos al nuevo marco del Espacio Europeo de Educación Superior (EEES). El EEES tiene como uno de los ejes de desarrollo la promoción de la calidad en las universidades.

Sin profesores competentes, la universidad no podría conquistar sus metas al no poder transferir de forma adecuada el conocimiento. Por tanto, es lógico considerar que el profesor desempeña un papel fundamental como referente en los nuevos diseños educativos de calidad universitaria [9]. Por ello, distintas universidades españolas utilizan diversos instrumentos para evaluar a los profesores a partir, fundamentalmente, de encuestas a los alumnos. La Universitat de Barcelona (UB), con sus 22 centros y cinco centros adscritos, en su proceso de mejora continua y con el objetivo de conocer el grado de satisfacción de los estudiantes acerca de la docencia de los profesores, puso en marcha un sistema de encuestas 'en papel' desde principio de los años noventa hasta el año 2010. Este modelo precisaba dos elementos que podían actuar tanto en sentido positivo como negativo: la voluntariedad de presentar las encuestas por parte de los profesores y el grado de cumplimiento por parte de los alumnos (el porcentaje de respuestas era del 40-50\%). Desde el año 2010-2011, en que la UB decidió adoptar el sistema de encuestas on line, el porcentaje de respuestas (por lo menos en la Facultad de Medicina) es del 0-7\%. Esta profunda reducción obedece, según palabras de los propios alumnos, al exceso de encuestas que se les solicita que cumplimenten, y a la poca o nula repercusión de sus resultados. Ambos tipos de encuestas (papel y on line) evaluaban a un determinado profesor de una asignatura en concreto. Desde una óptica distinta, la propuesta de este trabajo fue hacer una valoración genérica de la docencia clínica, sin valorar de forma individual a un determinado profesor o asignatura. Este modelo de encuesta se inició coincidiendo con el primer año en que se impartía la asignatura de 'Semiología General y Propedéutica Clínica' en el Grado de Medicina. Como quiera que la asignatura es semestral, cada año la asignatura se imparte dos veces y, en consecuencia, se presentan los resultados de cinco períodos. Si bien se han podido revisar artículos sobre docencia clínica, la mayoría de ellos se refieren a alumnos de medicina de los últimos cursos, a residentes o a especialistas en formación en especialidades preferentemente quirúrgicas. No hemos encontrado en la literatura médica consultada estudios similares con los que hacer comparaciones, pero si artículos relacionados con la práctica reflexiva [10] y sobre tecnologías educativas $[11,12]$.

\section{Materiales y métodos}

La 'Semiología General y Propedéutica Clínica' es una asignatura troncal fundamental, de 16 créditos ECTS, que se imparte en tercer curso del Grado de Medicina. Su programa incluye 55 clases magistrales y 32 seminarios. Por lo que respecta a la docencia clínica, el período es de siete semanas (28 días), de 8 a 13 horas. Los alumnos, en número de 4-8 por unidad, son adscritos a siete unidades u hospitales asociados: tres unidades de medicina interna del Hospital Clínic de Barcelona y los servicios de medicina interna de cuatro hospitales asociados. Estas unidades han sido nombradas adrede como A, B, C, D, E, F y G. El programa, los objetivos y los contenidos de la docencia clínica están consensuados previamente intentando ser lo más similares posibles, salvando algunos detalles propios de cada institución o unidad.

Se han analizado los cursos 2011-2012, 2012-2013 y el primer semestre del curso 2013-2014, repre- 
Tabla II. Hoja de respuestas de la encuesta ${ }^{\text {a. }}$

¿Hay objetivos planteados con ítems comprobables? (sí/no)

Dedicación del profesor clínico (de 1 a 5)

¿Se cumple el programa previsto? (de 1 a 5)

¿Quién asume la docencia?:

residentes (\%), profesor de la UB (\%), otros (\%)

¿El entorno es adecuado (médicos, enfermeras...)? (de 1 a 5)

¿El lugar es adecuado para la docencia clínica prevista? (de 1 a 5)

¿La duración de la docencia clínica es...?: corta, adecuada, excesiva

Valoración global (de 1a 5)

Aspectos que se echan en falta

Comentarios libres

a La puntuación oscila de 0 a 5, de menos a más.

sentando un total de cinco 'cursos', ya que la asignatura es semestral y se imparte dos veces en cada curso académico. A la llegada de los alumnos, se les informa de los objetivos de la estancia clínica, y al final del período, se les invita a cumplimentar una encuesta anónima y voluntaria en la que valoran distintos ítems.

Los objetivos fundamentales que se pretende que los alumnos consigan durante su estancia clínica se muestran en la tabla I.

Durante las siete semanas (28 días), cada uno de los alumnos debe cumplimentar un portafolio que contiene cinco historias clínicas completas, que entregan el último día de prácticas y es evaluado por el profesor clínico.

Una vez finalizadas las actividades docentes se solicita a los estudiantes su opinión mediante una encuesta, que completan de forma voluntaria y anónima, donde deben evaluar los ítems que se muestran en la tabla II. Como se puede observar, y excepto por dos cuestiones (dedicación del profesor clínico y porcentaje del tiempo), el resto de preguntas sólo contemplan datos generales sobre la estancia clínica.

\section{Análisis estadístico}

Los datos de la muestra se describen mediante recuentos y porcentajes, o mediante los tres cuartiles.
Tabla III. Respuestas por curso y por unidad docente $(n=477)$.

\begin{tabular}{|c|c|c|}
\hline \multirow{5}{*}{ Curso } & 2012 semestre 1 & $92(19,3 \%)$ \\
\hline & 2012 semestre 2 & $102(21,4 \%)$ \\
\hline & 2013 semestre 1 & $102(21,4 \%)$ \\
\hline & 2013 semestre 2 & $107(22,4 \%)$ \\
\hline & 2014 semestre 1 & $74(15,5 \%)$ \\
\hline \multirow{7}{*}{ Unidad } & $A$ & $141(29,6 \%)$ \\
\hline & $B$ & $84(17,6 \%)$ \\
\hline & $C$ & $72(15,1 \%)$ \\
\hline & $\mathrm{D}$ & $15(3,1 \%)$ \\
\hline & $E$ & $118(24,7 \%)$ \\
\hline & $\mathrm{F}$ & $38(8,0 \%)$ \\
\hline & $G$ & $9(1,9 \%)$ \\
\hline
\end{tabular}

Los cinco 'cursos' se compararon mediante pruebas de independencia basadas en el estadístico chi al cuadrado de Pearson (variables cualitativas) o mediante pruebas de Kruskall-Wallis (puntuaciones en escala de 1 a 5), utilizando un nivel de significación del 5\%, y sin realizar ajustes por multiplicidad, dado que los análisis se consideran exploratorios. Todos los análisis se efectuaron con el lenguaje R, versión 3.0.1.

\section{Resultados}

A lo largo de los cinco 'cursos' se obtuvieron 477 respuestas a las encuestas presentadas a los 502 alumnos que realizaron las prácticas de la asignatura (95\%). La distribución de respuestas por curso y por unidad docente se muestra en la tabla III.

Los resultados del análisis descriptivo de las respuestas obtenidas se muestran en la tabla IV.

La comparación de la valoración global entre los cinco cursos no detectó diferencias estadísticamente significativas $(p=0,658)$. Tampoco se detectaron diferencias entre los cinco cursos por lo que hace referencia a la dedicación del profesor $(p=0,730)$, a la adecuación del entorno ( $p=0,730)$ o a la existencia de objetivos verificables $(p=0,167)$.

Por el contrario, sí se detectaron diferencias estadísticamente significativas entre los cursos en cuanto a las percepciones del cumplimiento del progra- 
Figura. Diferencias entre los cinco cursos: a) Cumplimiento del programa; b) Adecuación del lugar; c) Duración de la docencia.

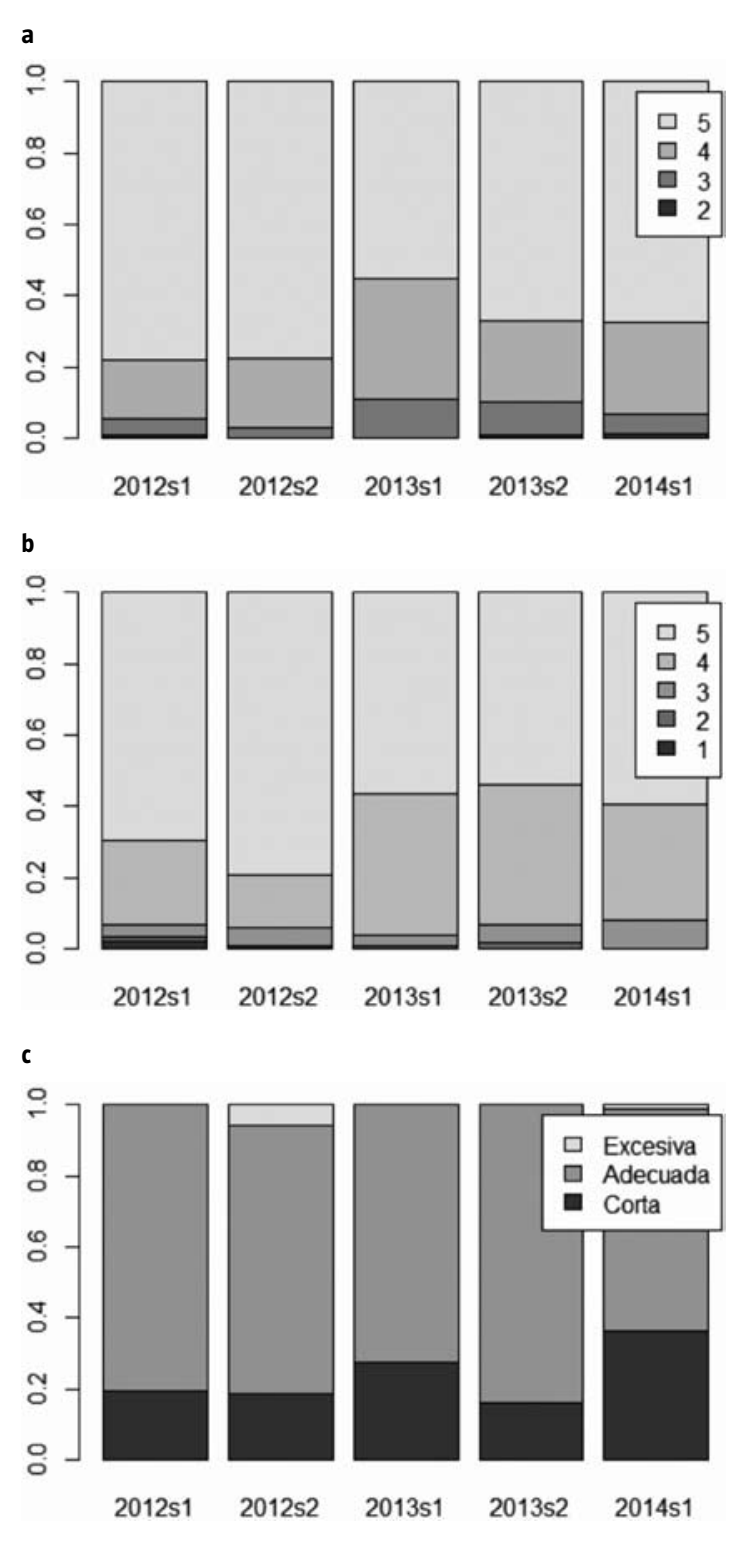

ma $(p=0,003)$, adecuación del lugar $(p=0,002)$ y duración del período de prácticas $(p<0,001)$. Las distribuciones de las respuestas a estas preguntas se muestran en la figura. Por lo que respecta a los comentarios libres, éstos se encontraron hasta en un $30 \%$ de las encuestas. Destacan los comentarios sobre el requerimiento de una mejor sistemática y organización de los seminarios de exploración física, y sobre la satisfacción de que con estas prácticas
Tabla IV. Descripción de los resultados de las encuestas $(n=477)$.

\begin{tabular}{|c|c|c|}
\hline & Sí & $429(89,9 \%)$ \\
\hline \multirow[t]{4}{*}{ Objetivos comprobables } & No & $30(6,3 \%)$ \\
\hline & No contesta & $18(3,8 \%)$ \\
\hline & Corta & $108(22,6 \%)$ \\
\hline & Adecuada & $357(74,8 \%)$ \\
\hline \multirow{3}{*}{ Duración } & Excesiva & $7(1,5 \%)$ \\
\hline & No contesta & $5(1,0 \%)$ \\
\hline & Profesor UB & $60 \%(40,80)$ \\
\hline \multirow[t]{2}{*}{ Asume la docencia ${ }^{a}$} & Residentes & $30 \%(10,50)$ \\
\hline & Otros & $10 \%(0,15)$ \\
\hline Cumplimiento del programa ${ }^{a}$ & & $5(4,5)$ \\
\hline Dedicación del profesor ${ }^{a}$ & & $5(4,5)$ \\
\hline Entorno adecuado ${ }^{a}$ & & $5(4,5)$ \\
\hline Lugar adecuado a & & $5(4,5)$ \\
\hline Valoración global ${ }^{a}$ & & $5(4,5)$ \\
\hline a Mediana (cuartil 1, cuartil 3) & & \\
\hline
\end{tabular}

clínicas los alumnos se habían reafirmado en que su verdadera vocación era ser médico.

\section{Discusión}

Una supuesta fortaleza del presente trabajo es que la docencia clínica recibida por los alumnos se basa en pacientes reales y en distintos dispositivos asistenciales. A nuestro entender, ello supera con creces cualquier aplicación telemática e incluso una prueba de evaluación de competencias objetiva estructurada [11,12]. Además, se sigue con fidelidad el principio de que nuestros futuros médicos deben prepararse de forma primordial para las enfermedades crónicas del siglo xxI en lugar de las enfermedades agudas del siglo xx [13]. Otra de las fortalezas del escenario del estudio es la ratio alumno/ profesor, ya que en estos momentos se acerca a 2-3 alumnos por profesor clínico.

Aunque se detectaron diferencias estadísticamente significativas entre los cursos en cuanto a la per- 
cepción del cumplimiento del programa $(p=0,003)$, la adecuación del lugar $(p=0,002)$ y la duración del período de prácticas $(p<0,001)$, éstas son probablemente poco relevantes desde un punto de vista práctico, al menos en el caso de la adecuación del lugar y del cumplimiento de objetivos, ya que parecen producirse a expensas de una distinta proporción de casos que asignan cuatro o cinco puntos a estas preguntas (Figura, a y b). En el caso de la duración de las prácticas, en el último curso (primer trimestre de 2014), aumenta la proporción de estudiantes que consideran que las prácticas son demasiado cortas. Al no identificarse ninguna razón objetiva que explique este cambio de percepción, se atribuye a un efecto del grupo de estudiantes, aunque en los comentarios libres que podían hacer los estudiantes, la razón que aducían en cuanto a que su estancia les había resultado corta era que la asignatura les permitía por primera vez tener contacto con los pacientes (en los cursos anteriores, e incluso en algunas asignaturas de tercero, las prácticas no las realizan con pacientes), por lo que les resultaba altamente estimulante enfrentarse a lo que será su futuro como médicos. Como muy bien describían Baños et al [1], el contacto precoz del estudiante de medicina con la realidad asistencial podía tener efectos beneficiosos sobre la motivación, el conocimiento de la relación médico-paciente y la aceptación de la importancia de las materias médicas básicas.

En cuanto a la responsabilidad de la docencia por parte del profesor de la UB, que en nuestros resultados se comprueba que es de un 60\% (mediana), ello lógicamente implica que en el $40 \%$ restante la docencia es impartida por residentes u otro personal sanitario. Aunque este dato puede implicar una lectura negativa por lo que se refiere a la responsabilidad del profesor, no es menos cierto que el alumno se beneficia también de las enseñanzas de los residentes, en la elaboración de las historias clínicas, en la toma de decisiones clínicas, en la gestión de las interconsultas con los especialistas, etc. Por fin, el hecho de que el porcentaje de respuestas de las encuestas supere el 95\%, creemos que es de suma importancia. Los estudiantes valoran de for- ma muy positiva el poco tiempo que les lleva cumplimentarlas y, en particular, que del análisis de los datos obtenidos se puedan derivar consecuencias positivas en aras a mejorar la docencia clínica. En este sentido, algunos resultados parciales del estudio se presentaron en las Jornadas de Docencia de la Facultad de Medicina en 2012, a las que asisten numerosos estudiantes.

En resumen, y en términos generales, la experiencia de nuestros alumnos sobre la docencia clínica fue altamente satisfactoria, sin mostrar variaciones relevantes en los cinco cursos analizados. Ello sin duda representa un estímulo para que profesores y alumnos sigan en la misma línea y para mejorar si cabe aquellos aspectos susceptibles de modificarse.

\section{Bibliografía}

1. Baños JE, Sentí M, Miralles R. Contacto precoz con la realidad asistencial: una experiencia piloto en medicina. Educ Med 2011; 14: 39-47.

2. Felman KA. Consistency and variability among college students in rating their teachers and courses: a review and analysis. Research in Higher Education 1977; 6: 223-7.

3. Dawoud M. Une grille d'évaluation de la compétence du professeur d'université. Rev Psychol Appl 1983; 33: 17-31.

4. Miller AH. Student assessment of teaching in higher education. Higher Education 1983; 17: 1-13.

5. Miron M. Students 'evaluation and instructors' self-evaluation of university instruction. Higher Education 1988; 17: 175-81.

6. Rushton JP, Murray HG. On the assessment of teaching effectiveness in British universities. Bull Br Psychol Soc 1985; 3: 361-5.

7. Watkins D, Marsh HW, Young D. Evaluating tertiary teaching: a New Zealand perspective. Teaching \& Teacher Education 1987; 3: 41-53.

8. Fernández J, Mateo MA, Muñiz J. Valoración por parte del profesorado de la evaluación docente realizada por los alumnos. Psicothema 1996; 8: 167-72.

9. Leiva JJ. La participación del alumnado en la evaluación docente: reflexiones para el debate en el contexto universitario. Revista de Formación Universitaria 2011; 4: 170-6.

10. Chaffey LJ, Janet de Leeuw EJ, Finnigan GA. Facilitating student's reflective practice in a medical course: literature review. Educ Health 2012; 25: 198-203.

11. Han H, Resch DS, Kovach RA. Educational technology in medical education. Teach Learn Med 2013; 25: S39-43.

12. Burgess AW, McGregor DM, Mellis CM. Applying established guidelines to team-based learning programs in medical schools: a systematic review. Acad Med 2014; 89: 678-88.

13. Lucey CR. Medical education. Part of the problem and part of the solution. JAMA Intern Med 2013; 173: 1639-43. 